\title{
VARIABLE GAP UNDULATOR FOR 1.5-48 KEV FREE ELECTRON LASER AT LINAC COHERENT LIGHT SOURCE*
}

\author{
C. Pellegrini, UCLA, Los Angeles, CA, USA \\ J. Wu, SLAC, Menlo Park, CA, USA
}

\begin{abstract}
We study the feasibility of generating femtosecond duration Free-Electron Laser with a variable photon energy from 1.5 to $48 \mathrm{keV}$, using an electron bunch with the same characteristics of the LINAC Coherent Light Source (LCLS) bunch, and a planar undulator with additional focusing. We assume that the electron bunch energy can be changed, and the undulator has a variable gap, allowing a variable undulator parameter. It is assumed to be operated in an ultra-low charge and ultra-short pulse regime.
\end{abstract}

\section{INTRODUCTION}

The success of the LINAC Coherent Light Source (LCLS) [1] motivates an extension of the capacity, capabilities, and quality of this revolutionary new light source. Here, we study the possibility of generating hard x-ray FEL at LCLS with a wide tunable photon energy of 1.5 - $48 \mathrm{keV}$. A planar undulator with additional focusing is assumed. To achieve the wide tunable range, the electron beam energy is changed between 4.6 and $13.8 \mathrm{GeV}$, and the undulator, with a $2.5 \mathrm{~cm}$ period, variable gap is also varied accordingly to allow the undulator parameter to be changed to satisfy the resonant condition. Based on analytical calculation, we study the requirements on the electron bunch parameters and the FEL performance, optimizing the system parameters. Detailed numerical simulation using GENESIS [2] is done with the optimized parameter set.

\section{ELECTRON BUNCH PARAMETERS}

We explore the parameter space first with analytical scalings. We evaluate the characteristics of an X-ray FEL, like gain length, pulse duration, power, as a function of the radiation wavelength, beam energy, electron beam charge, and peak current, using Ming Xie code [3].

The normalized emittance scales like the cubic root of the charge, in the low charge regime, where the thermal emittance dominates. If the contributions from RF curvature and space charge cannot be neglected the transverse normalized emittance depends on charge as [4]:

$$
\begin{aligned}
\varepsilon_{N} & =1.5 \times 10^{-6}\left(0.111 \times 10^{6} Q^{2 / 3}+0.18 \times 10^{12} Q^{4 / 3}\right. \\
& \left.+0.18 \times 10^{24} Q^{8 / 3}\right)^{1 / 2}
\end{aligned}
$$

where $Q$ is in units of Coulomb and $\varepsilon_{N}$ in m-rad. With this scaling, the transverse normalized emittance as a function of electron bunch charge for low-charge region

*Work supported in part by the DOE Contract DE-AC0276SF00515. This work was performed in support of the LCLS project at SLAC. can be estimated. Here, we consider the case of $Q=10$ $\mathrm{pC}$ with $\varepsilon_{N}=0.11 \mathrm{~m}$-mrad.

Since the peak current and the bunch length depend on the photo-injector and bunch compression, the situation in this case is more complicated. We scale the bunch length at the photo-injector exit as the charge to the power $1 / 3$ and assume also that we use velocity bunching. For the low-charge range in which we are interested, as mentioned above, the electron bunch rms length as a function of charge is assumed to be $\sigma_{L}=0.03 Q^{1 / 3}$, where $\sigma_{L}$ is in units of meters and $Q$ in Coulomb. Notice that, for $Q=10 \mathrm{pC}, \sigma_{L} \approx 6.5 \mu \mathrm{m}$, which gives a peak current ${ }^{1}$ of $I_{p k}=185 \mathrm{~A}$. Here, we assume that we require the final peak current to be $2 \mathrm{kA}$ after compression. For the case of $Q=10 \mathrm{pC}$, the magnetic compression factor is about 11 with the initial peak current of about $185 \mathrm{~A}$. Notice that for this case of $Q=10 \mathrm{pC}$, the final $I_{p k}=2$ $\mathrm{kA}$ sets the electron bunch final rms length of $0.6 \mu \mathrm{m}$, i.e., rms temporal duration of $2 \mathrm{fs}$. This should make the final FEL approaching the single spike operation mode at $\lambda_{r}=1 \mathrm{~nm}$.

\section{FEL PERFORMANCE}

We briefly review the key physics parameters which we need to consider, before optimizing the system based on these considerations. The FEL parameter [5] is defined as

$$
\rho=\left(1 / 2 \gamma_{0}\right)\left\{\left(I_{p k} / I_{A}\right)\left[\left(K^{2}[J J]^{2}\right) /\left(2 k_{w}^{2} \sigma_{x} \sigma_{y}\right)\right]\right\}^{1 / 3},
$$

where $\gamma_{0}=\left[\left(1+K^{2} / 2\right) \lambda_{w} /\left(2 \lambda_{r}\right)\right]^{1 / 2}$ is the Lorentz factor of the electron resonant energy with $\lambda_{r}$ the resonant FEL radiation wavelength; $K \approx 93.4 B_{w} \lambda_{w}$ where $B_{w}$ is the undulator peak field in Teslas and $\lambda_{w}$ the undulator period in meters; $[J J]=J_{0}[\xi]-J_{1}[\xi]$, with $\xi \equiv a_{w}^{2} /\left[2\left(1+a_{w}^{2}\right)\right]$ and $a_{w}=K / \sqrt{2}, J_{0}$ and $J_{1}$ being the $0^{t h}$-order and $1^{\text {st }}$-order Bessel functions; $k_{w}=2 \pi / \lambda_{w}$; $\sigma_{x(, y)}=\sqrt{\varepsilon_{N} \beta_{x(, y)} / \gamma_{0}}$ is the electron beam transverse

\footnotetext{
1 The definition of the peak current is $I_{p k} \equiv Q c /\left(\sqrt{2 \pi} \sigma_{L}\right)$, where $c$ is the speed of light in vacuum.
} 
$x(, y)$ rms size; $I_{p k}$ is the peak current; and $I_{A} \equiv$ $4 \pi \epsilon_{0} m c^{3} / e \approx 17045 \mathrm{Amp}$, the Alfvèn current. With this FEL parameter $\rho$, which characterizes the coupling efficiency of the electron bunch and the FEL, the 1-D power gain length is readily defined as $L_{G}^{1 D} \equiv \lambda_{w} /(4 \sqrt{3} \pi \rho)$. We optimize the beta function assuming that an external focusing (e.g., FODO cell lattice) is applied. It has been shown from rigorous analysis [6] that the FEL gain length can be expressed by a universal scaling function $L_{G}^{1 D} / L_{G}=F\left(\eta_{d}, \eta_{\varepsilon}, \eta_{\delta}\right)$, where $\eta_{d}=L_{G}^{1 D} / Z_{R}$ characterizes the diffraction effect with $Z_{R}=4 \pi \sigma_{x}^{2} / \lambda_{r}$ being the Rayleigh range; $\eta_{\varepsilon}=\left(L_{G}^{1 D} / \beta\right)\left[4 \pi \varepsilon_{N} /\left(\gamma_{0} \lambda_{r}\right)\right]$ characterizes the emittance effect; and $\eta_{\delta}=4 \pi \sigma_{\delta} L_{G}^{1 D} / \lambda_{w}$ characterizes the chromatic effect, where $\delta=\left(\gamma-\gamma_{0}\right) / \gamma_{0}$ is the relative energy deviation. According to Ref. [3], the universal function $F\left(\eta_{d}, \eta_{\varepsilon}, \eta_{\delta}\right)$ can be fitted into a 19-parameter function $\eta$, so that $L_{G}^{1 D} / L_{G}=1 /(1+\eta)$. The saturation power is fitted empirically as $P_{\text {sat }} \approx$ $1.6 \rho\left(L_{G}^{1 D} / L_{G}\right)^{2} \gamma_{0} m_{e} c^{2} I_{p k}$.

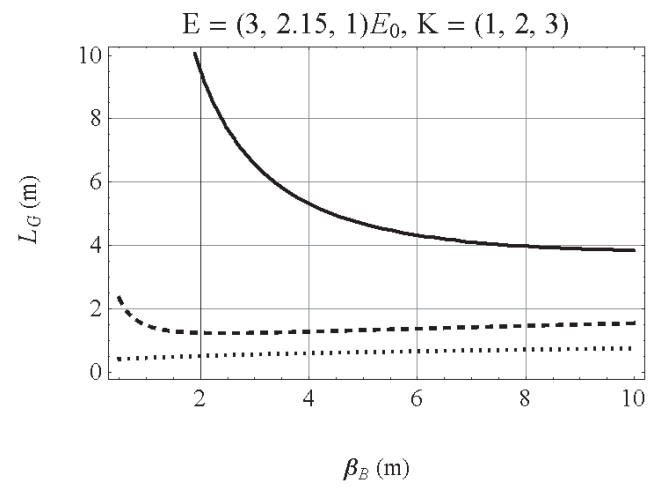

FIG. 1: FEL gain length as a function of $\beta$-function for various $E$ and $K=1$ (solid curve), 2 (dashed curve), and 3 (dotted curve).

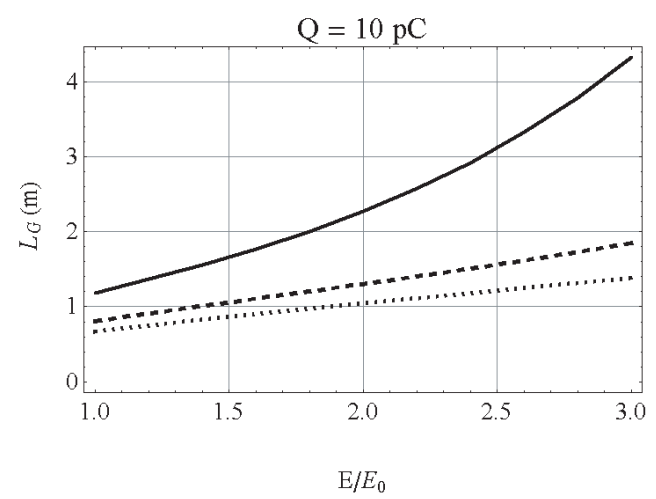

FIG. 2: FEL gain length for fixed $\beta_{B}=6 \mathrm{~m}$, but with various $E$ and $K=1$ (solid curve), 2 (dashed curve), and 3 (dotted curve).

For a SASE FEL, coherent spikes develop during the FEL process. The FWHM duration of the coherent spike

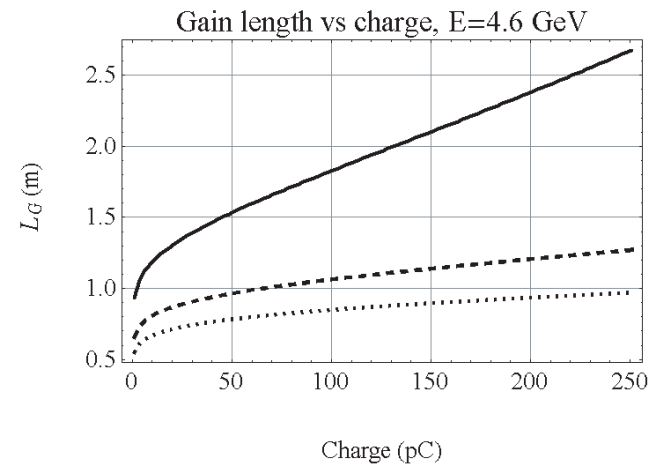

FIG. 3: FEL gain length for fixed $\beta_{B}=6 \mathrm{~m}$ and fixed energy $E_{0}=4.6 \mathrm{GeV}$, but with various charge $Q$ and $K=1$ (solid curve), 2 (dashed curve), and 3 (dotted curve).

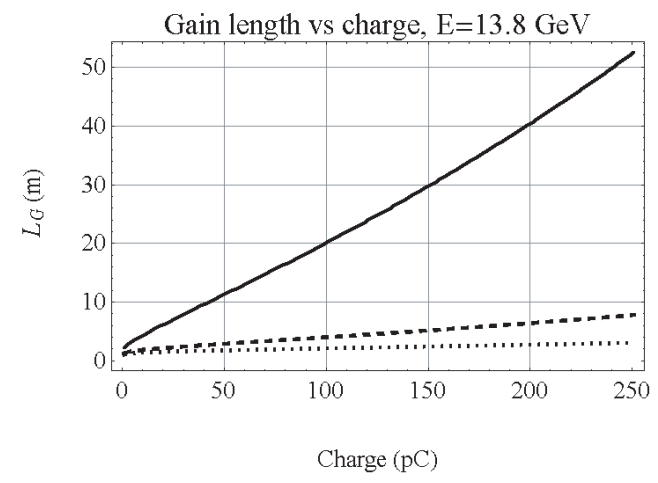

FIG. 4: FEL gain length for fixed $\beta_{B}=6 \mathrm{~m}$ and fixed energy $E_{0}=13.8 \mathrm{GeV}$, but with various charge $Q$ and $K=1$ (solid curve), 2 (dashed curve), and 3 (dotted curve).

is related to the FEL bandwidth [7]

$$
L_{\text {spike }}(z)=c \sqrt{\pi} / \sigma_{\omega}(z),
$$

where $\sigma_{\omega}(z)=\omega_{r} \sqrt{3 \sqrt{3} \rho /\left(k_{w} z\right)}$, is the FEL rms bandwidth as a function of the $z$-coordinate in the undulator. The total number of spikes is $N_{\text {spike }}=\sigma_{L} / L_{\text {spike }}$, for an

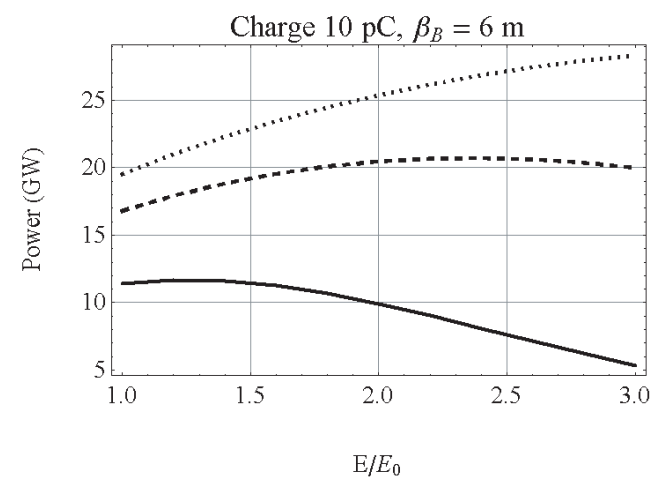

FIG. 5: FEL saturation power for fixed $\beta_{B}=6 \mathrm{~m}$ and fixed charge $Q=10 \mathrm{pC}$, but with various $E$ and $K=1$ (solid curve), 2 (dashed curve), and 3 (dotted curve). 


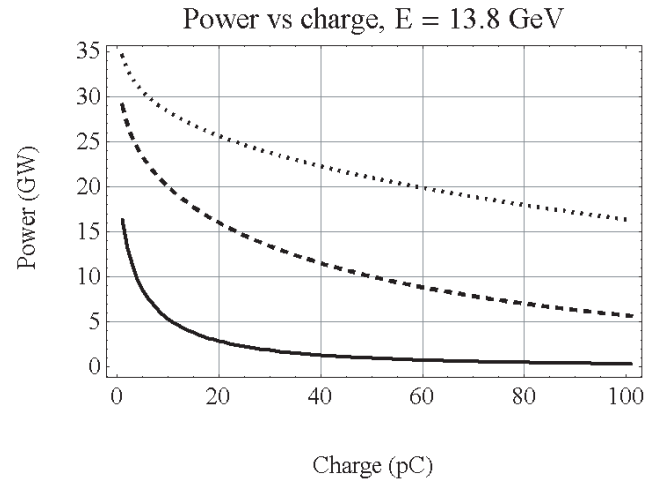

FIG. 6: FEL saturation power for fixed $\beta_{B}=6 \mathrm{~m}$ and fixed $E=13.8 \mathrm{GeV}$, but with various charge $Q$ and $K=1$ (solid curve), 2 (dashed curve), and 3 (dotted curve).

electron bunch having a Gaussian temporal distribution with rms length of $\sigma_{L}$. Notice that, due to the highly nonlinear dependence of the FEL gain on the current, for a temporal Gaussian distribution, the effective length is $\sigma_{L}$.

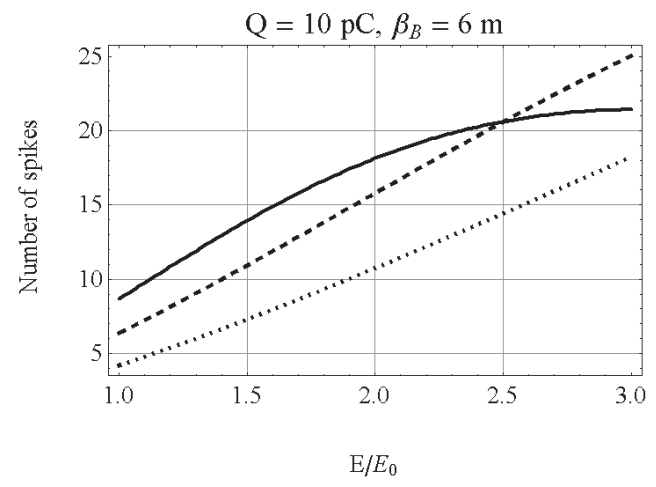

FIG. 7: Coherent spikes \# vs. beam energy for a fixed charge of $10 \mathrm{pC}$ and various $K=1$ (solid curve), 2 (dashed curve), and 3 (dotted curve).

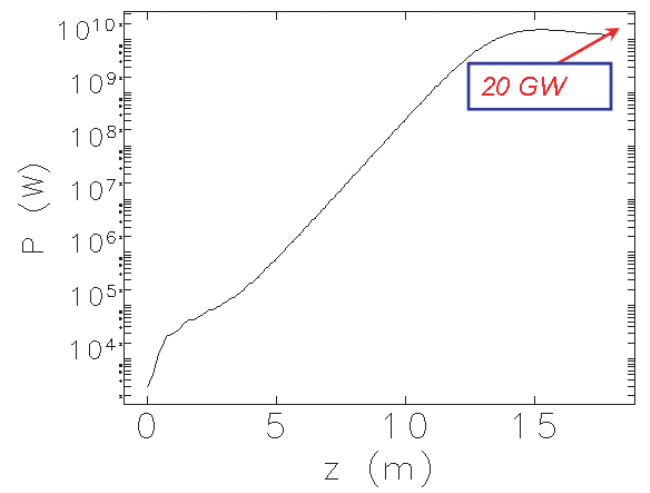

FIG. 8: GENESIS simulation of the FEL power gain curve for the $1.5 \mathrm{keV}$ FEL case.

\section{A. Optimization}

Here, we use the notation $E_{0}=4.6 \mathrm{GeV}$ and the undulator period $\lambda_{w}=0.025 \mathrm{~m}$. We vary the electron energy $E$ and the undulator parameter $K$ to find a reasonable parameter set. We first find what is the optimum value of the focusing in the undulator. Since the gain length would be the longer for shorter wavelength, we optimize the focusing strength with emphasis on the short wavelength end. To do this we consider the case of the shortest wavelength, with $K=1$ and $E=13.8 \mathrm{GeV}$, and plot the gain length as a function of the focusing strength. This case corresponds to the FEL photon energy of $48 \mathrm{keV}$ (FEL wavelength of $\lambda_{r}=0.26 \AA$ ), and is shown as the solid curve in Fig. 1. In the same Fig. 1, we also show two other cases for FEL with photon energy of $1.5 \mathrm{keV}$ (FEL wavelength of $\lambda_{r}=8.5 \AA$ ) (dotted curve) and 12.4 $\mathrm{keV}$ (FEL wavelength of $\lambda_{r}=1.0 \AA$ ) (dashed curve) as comparison. In the following calculations, we will choose $\beta_{B}=6 \mathrm{~m}$ to cover all the cases.

For this chosen $\beta_{B}=6 \mathrm{~m}$, we plot in Fig. 2, the gain length as a function of energy for $K=1$ (solid curve), 2 (dashed curve), and 3 (dotted curve). All the calculations are for charge $Q=10 \mathrm{pC}$ and with the final peak current of $I_{p k}=2 \mathrm{kA}$. It is seen that for the shortest wavelength case, the gain length is slightly longer than $4 \mathrm{~m}$.

In Fig. 3 we show the gain length as a function of electron bunch charge $Q$ for $K=1$ (solid curve), 2 (dashed curve), and 3 (dotted curve). The plot in Fig. 3 shows clearly that at minimum beam energy, corresponding to wavelength between 8 and $2 \AA$ as $K$ is decreased from 3 to 1 , the gain length remains always within good values, less than $3 \mathrm{~m}$. We repeat now the evaluation of the gain length as a function of charge at the maximum beam energy of $E=13.8 \mathrm{GeV}$. Figure 4 shows that at the maximum beam energy and $K=1$, only small values of charge (up to $15 \mathrm{pC}$ ) can be used for a reasonably short gain length. The FEL saturation power as a function of $E$ and $K$ at a fixed charge of $10 \mathrm{pC}$ is plotted in Fig. 5, which shows that with these parameters, the FEL peak power can always be larger than GW. For a more close look at the high energy end, we plot in Fig. 6, the FEL saturation power as a function of charge for various $K=$ 1 (solid curve), 2 (dashed curve), and 3 (dotted curve). Again, to get GW level FEL power, a charge as low as $15 \mathrm{pC}$ is necessary for $48 \mathrm{keV}$ FEL.

\section{B. Coherence}

Due to the fact that the SASE FEL starts from shotnoise, the temporal coherence is developed purely due to the slippage of the photon over the electrons. For a fixed charge of $10 \mathrm{pC}$, to generate $1.5-48 \mathrm{keV}$ FEL with various undulator parameter and electron energy, the coherent spike can be as long as a few tens of nm. For charge of $10 \mathrm{pC}$, and final peak current of $I_{p k}=2 \mathrm{kA}$, the electron bunch length is on the order of $1 \mu \mathrm{m}$. So, for the low 

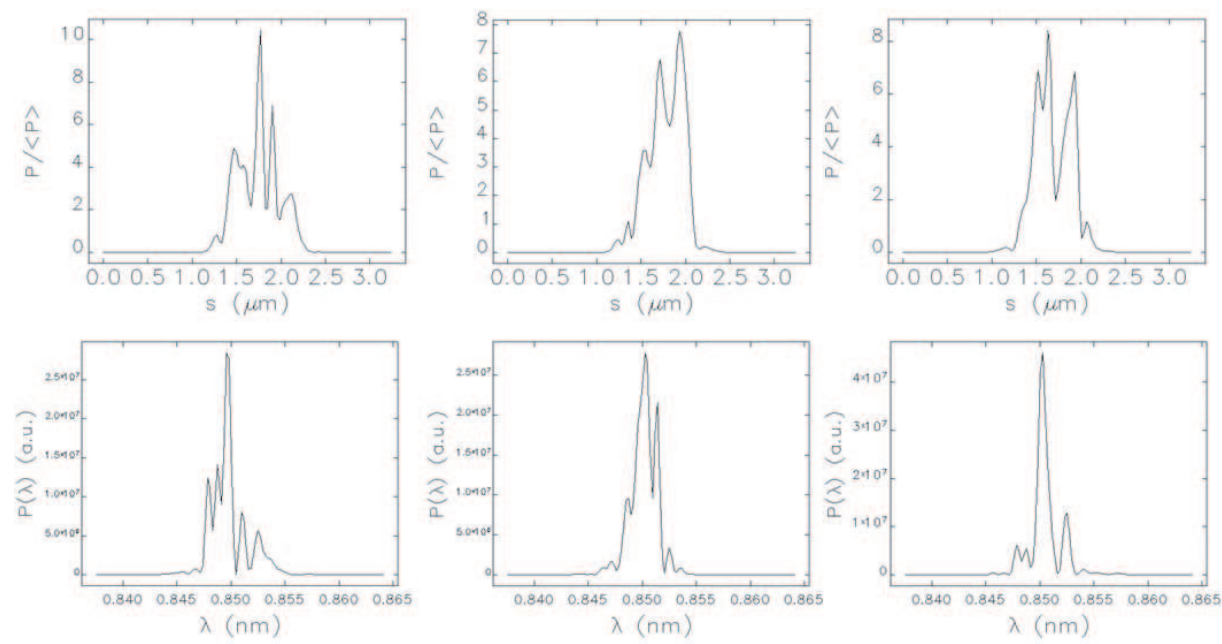

FIG. 9: GENESIS simulation of the FEL (1.5 keV) pulse at $15 \mathrm{~m}$ into the undulator showing just a few spikes.

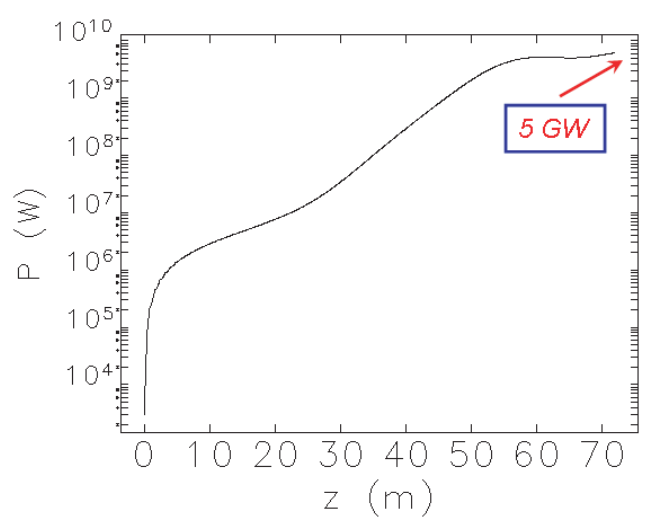

FIG. 10: GENESIS simulation of the FEL power gain curve for the $48 \mathrm{keV}$ FEL case.

energy FEL case, there will be a few to a few tens spikes in the pulse. This is shown in Fig. 7.

As a summary, in Table I, we show two extreme cases: the low energy $1.5 \mathrm{keV}$ and the high energy $48 \mathrm{keV}$ FEL.

TABLE I: Two Extreme Cases (1.5 keV and $48 \mathrm{keV}$ FEL).

\begin{tabular}{|l|c|c|c|}
\hline \hline Parameter (Symbol) & $1.5 \mathrm{keV}$ & $48 \mathrm{keV}$ & Units \\
\hline Electron energy $(\mathrm{E})$ & 4.599 & 13.797 & $\mathrm{GeV}$ \\
FEL wavelength $\left(\lambda_{r}\right)$ & 8.488 & 0.257 & $\AA$ \\
Pierce parameter $(\rho)$ & 2.23 & 0.58 & $10^{-3}$ \\
Gain length $\left(L_{G}\right)$ & 0.668 & 4.325 & $\mathrm{~m}$ \\
Saturation power $\left(P_{\text {sat }}\right)$ & 19.5 & 5.4 & $\mathrm{GW}$ \\
Coherent photon \# $(N)$ & 166.5 & 1.4 & $10^{9}$ \\
Spike length $\left(L_{\text {coop }}\right)$ & 142.6 & 27.6 & $\mathrm{~nm}$ \\
Spikes \# $\left(N_{\text {spike }}\right)$ & 4.2 & 21.4 & \\
\hline
\end{tabular}

\section{GENESIS SIMULATION}

In above, we discussed the overall FEL performance using analytical formulae. Here, we choose three FEL cases, with 1.5, 12.4, and $48 \mathrm{keV}$ FEL resonant energy, and evaluate in detail the FEL characteristics using GENESIS. In Table II we show the key parameters used in the simulation.

First we consider the $1.5 \mathrm{keV}$ FEL case. In Fig. 8, we show the FEL power gain curve. It is seen that around 15 meters into the undulator, the FEL starts to saturate, with a saturation power around $20 \mathrm{GW}$. As expected, around saturation there are only a few spikes as in Fig. 9, where the FEL pulse temporal profile and spectrum are taken at 15 meters into the undulator. With three different initial random seeds, the final FEL pulse contains a few spikes as in the upper row of Fig. 9. The corresponding spectrum for each case is shown as the lower row in Fig. 9. The results for the $48 \mathrm{keV}$ case are in Fig. 10 showing a saturation length of about $55 \mathrm{~m}$ and a saturation power of $5 \mathrm{GW}$.

\section{CONCLUSIONS}

We study the feasibility of a tunable, short pulse, Xray FEL with photon energy from 1.5 to $48 \mathrm{keV}$, using an electron beam like the one in the LCLS and a $2.5 \mathrm{~cm}$ period, variable gap, planar undulator. The beam energy changes from 4.6 to $13.8 \mathrm{GeV}$, the electorn charge is kept at $10 \mathrm{pC}$, and the undulator parameter varies from 1 to 3 . The undulator length needed to saturate the $48 \mathrm{keV}$ FEL is about $55 \mathrm{~m}$, with a peak power around $5 \mathrm{GW}$. At longer wavelength the saturation length is as short as $15 \mathrm{~m}$, and the peak power around $20 \mathrm{GW}$. The results from the analytical models and the GENESIS simulations show that the system is feasible. The large wavelength range, full tunability and short, few femtosecond pulses, 
TABLE II: Parameters and Performance for the Three FEL Cases: 1.5, 12.4, and 48 keV FEL with GENESIS Simulation.

\begin{tabular}{|l|c|c|c|c|c|}
\hline \hline Parameter & Symbol & $1.5 \mathrm{keV}$ FEL & $12.4 \mathrm{keV}$ FEL & $48 \mathrm{keV}$ FEL & Units \\
\hline Electron bunch centroid energy & $\mathrm{E}$ & 4.6 & 9.9 & 13.8 & $\mathrm{GeV}$ \\
Electron bunch slice relative energy spread & $\sigma_{\delta}$ & 1.0 & 0.46 & 0.33 & $10^{-4}$ \\
FEL wavelength & $\lambda_{r}$ & 8.5 & 1.0 & 0.26 & $A$ \\
FEL power $e$-folding length & $L_{G}$ & 0.8 & 1.2 & 3.0 & $\mathrm{~m}$ \\
FEL saturation power & $P_{\text {sat }}$ & 20 & 20 & 5 & $\mathrm{GW}$ \\
Number of photon per pulse & $N$ & 20 & 5.3 & 0.3 & $10^{10}$ \\
\hline
\end{tabular}

together with the large peak power, would provide a pow- $\quad$ erful research tool.

[1] P. Emma et al., Nature Photonics, 2010 (published online: 1 Aug 2010 - DOI: 10.1038/NPHOTON.2010.176).

[2] S. Reiche, NIMA 429, 243 (1999).

[3] M. Xie, PAC95, p. 183, 1995.

[4] J.B. Rosenzweig and E. Colby, in AIP Conf. Proc. Vol. 335, p. 724 (1995).
[5] R. Bonifacio, C. Pellegrini, and L.M. Narducci, Opt. Commun., 50, 373 (1984).

[6] L.-H. Yu et al., Phys. Rev. Lett., 64, 3011 (1990); Y.H. Chin et al., Phys. Rev. A 46, 6662 (1992).

[7] E.L. Saldin et al., Opt. Commun., 148, 383 (1998). 